\title{
Aging-Dependent Alterations in Synaptic Plasticity and Memory in Mice That Overexpress Extracellular Superoxide Dismutase
}

\author{
Daoying Hu, ${ }^{1}$ Faridis Serrano, ${ }^{1}$ Tim D. Oury, ${ }^{3}$ and Eric Klann ${ }^{1,2}$ \\ Departments of ${ }^{1}$ Molecular Physiology and Biophysics and ${ }^{2}$ Neuroscience, Baylor College of Medicine, Houston, Texas 77030, and ${ }^{3}$ Department of \\ Pathology, University of Pittsburgh Medical Center, Pittsburgh, Pennsylvania 15261
}

Oxidative damage caused by reactive oxygen species (ROS) has been proposed to be critically involved in several pathological manifestations of aging, including cognitive dysfunction. ROS, including superoxide, are generally considered as neurotoxic molecules whose effects can be alleviated by antioxidant enzymes. However, ROS also are known to be necessary components of the signal transduction cascades underlying normal synaptic plasticity. Therefore, we reasoned that the role that ROS and antioxidant enzymes play in modulating neuronal processes varies over the lifespan of an animal. We examined hippocampal long-term potentiation (LTP) and memoryrelated behavioral performance in transgenic mice overexpressing extracellular superoxide dismutase (EC-SOD) and their wild-type littermates at different ages. We found that aged EC-SOD transgenic mice exhibited enhanced hippocampal LTP, better cerebellumdependent motor learning, and better hippocampus-dependent spatial learning compared with their wild-type littermates. We also found that EC-SOD overexpression impaired contextual learning, but the impairment was decreased in the aged transgenic mice. At the molecular level, aged EC-SOD transgenic mice had lower superoxide levels, a decrease in protein carbonyl levels, and a decrease in p38 and extracellular signal-regulated kinase 2 phosphorylation compared with aged wild-type mice. Our findings suggest that elevated levels of superoxide contribute to aging-related impairments in hippocampal LTP and memory, and that these impairments can be alleviated by overexpression of EC-SOD. We conclude that there is an age-dependent alteration in the role of superoxide in modulating synaptic plasticity and learning and memory.

Key words: EC-SOD; superoxide; aging; oxidative stress; learning and memory; long-term potentiation

\section{Introduction}

Aging is accompanied by the loss of cognitive function, especially in certain types of learning and memory. Oxidative damage produced by excessive reactive oxygen species (ROS) is thought to underlie aging-related cognitive impairment and neurodegeneration. Excessive ROS can cause random cellular damage via modification of lipids, protein, and DNA. The brain is particularly vulnerable to oxidative stress because it consumes large amounts of oxygen and has abundant lipid content but a relative paucity of antioxidant levels compared with other organs (Halliwell, 1992). A large body of evidence has linked phenotypes associated with age to the accumulation of oxidative damage. For instance, age-related loss of cognitive and motor function is associated with oxidative damage of proteins and nucleic acids in the brain (Forster et al., 1996; Nicolle et al., 2001).

Superoxide dismutases (SODs) scavenge superoxide and are a

Received Aug. 26, 2005; revised Feb. 13, 2006; accepted March 3, 2006.

This work was supported by National Institutes of Health Grants NS034007 and NS047384 and the Cynthia and George Mitchell Foundation (E.K.). We thank Dr. Lingfei Hou and Kenneth T. Kishida for technical assistance and helpful suggestions.

Correspondence should be addressed to Dr. Eric Klann, Department of Molecular Physiology and Biophysics, Baylor College of Medicine, One Baylor Plaza, BCM 335, Houston, TX 77030. E-mail: eklann@bcm.tmc.edu. DOI:10.1523/JNEUROSCI.5566-05.2006

Copyright $\odot 2006$ Society for Neuroscience $\quad$ 0270-6474/06/263933-09\$15.00/0 major part of the cellular armamentarium against excess levels of ROS. Genetic and pharmacological manipulation of SOD activity has a profound effect on the aging and aging-related processes in Drosophila and mice. Overexpression of mitochondrial SOD (referred to as either SOD-2 or Mn-SOD) was shown to extend the lifespan of flies (Sun et al., 2002) and to attenuate drug-induced neurotoxicity in mice (Klivenyi et al., 1998). Overexpression of cytoplasmic SOD (referred to as either SOD-1 or Cu/Zn-SOD) in mice was shown to improve age-related impairments in longterm potentiation (LTP) (Kamsler and Segal, 2003), a cellular model of memory, and overexpression of extracellular SOD (ECSOD) has been shown to result in improved spatial memory in aged mice indicated by superior radial-arm maze choice accuracy (Levin et al., 2005). Moreover, chronic treatment of mice with synthetic SOD/catalase mimetics reverses hippocampusdependent learning deficits in aged mice (Liu et al., 2003). Thus, increasing the levels of SOD can improve aging-related deficits in both flies and mice.

Although ROS can cause damage to cells, they also have normal regulatory functions in biological processes (for review, see Esposito et al., 2004). One type of process that is tightly regulated by ROS is LTP (Knapp and Klann, 2002a). Cell-permeable superoxide scavengers block LTP (Klann, 1998), and cell-impermeable superoxide scavengers strongly attenuate LTP (Klann et al., 
1998). In addition, hippocampal slices from transgenic mice that overexpress either SOD-1 or EC-SOD (Gahtan et al., 1998; Thiels et al., 2000) exhibit deficient LTP. Thus, in contrast to their harmful role in contributing to the pathophysiology of aging, ROS play an important role in physiological processes such as synaptic plasticity.

These previous findings suggest that ROS have a dual role in regulating synaptic plasticity and memory that is age dependent. To test this notion, we used transgenic mice that overexpress human EC-SOD and examined hippocampal synaptic plasticity and memory-related behavioral performance at different ages. In addition, we also examined in vivo brain superoxide levels, brain protein oxidation, and the levels of active mitogen-activated protein kinases (MAPKs). The findings presented herein indicate that overexpression of EC-SOD protects mice from aging-related impairments in synaptic plasticity and memory by reducing oxidative stress.

\section{Materials and Methods}

EC-SOD transgenic mice. Transgenic mice were generated as described previously (Oury et al., 1992; Thiels et al., 2000). All mice were housed in the Transgenic Mouse Facility of the Baylor College of Medicine, compliant with the NIH Guide for Care and Use of Laboratory Animals. The facility is kept on a $12 \mathrm{~h}$ light/dark cycle, with regular feeding and cagecleaning schedule. Heterozygote mice expressing human EC-SOD were compared with wild-type mice from the same litter for all experiments.

Western blot analysis. Equivalent amounts of protein for each sample were resolved by SDS-PAGE and transferred to polyvinylidene difluoride (PVDF) membranes. The PVDF membranes were blocked in $5 \%$ nonfat dry milk for $1 \mathrm{~h}$ in Tris-buffered saline containing Tween 20 and then incubated with the specific antibody of interest [SOD-1 antibody, 1:500; SOD-2 antibody, 1:1000; catalase antibody, 1:10,000; phosphorylated (phospho)-p38 antibody, 1:1000; phospho-extracellular signalregulated kinase (ERK) antibody, 1:5000] overnight at $4^{\circ} \mathrm{C}$, followed by incubation with horseradish peroxidase-linked goat anti-rabbit IgG (1: $10,000)$ and developed using enhanced chemiluminescence. The blots used to detect phospho-p38 and phospho-ERK were incubated in stripping buffer $(25 \mathrm{~mm}$ glycine- $\mathrm{HCl}, \mathrm{pH} 2.0,1 \% \mathrm{SDS})$ for $1 \mathrm{~h}$ at $55-60^{\circ} \mathrm{C}$ and washed with Tris-buffered saline containing Tween 20 three times, 15 min for each wash. The stripped blots were incubated with an antibody directed against total levels of p38 or ERK (p38 antibody, 1:1000; ERK antibody, 1:5000). The SOD-1 and SOD-2 antibodies were purchased from Upstate (Charlottesville, VA), the catalase antibody was purchased from Chemicon (Temecula, CA), the phospho-p38 and p38 antibodies were purchased from Cell Signaling Technology (Beverly, MA), and the phospho-ERK and ERK antibodies were purchased from Promega (Madison, WI). The horseradish peroxidase-linked goat anti-rabbit IgG was obtained from Promega. Densitometric analysis of phosphoimmunoreactivity and total p38 or ERK2 immunoreactivity for each protein was conducted using Scion Image software (Scion, Frederick, MD). Phosphorylated immunoreactivity was normalized to total immunoreactivity first and then normalized to the young, wild-type values. The statistical analysis described in the table and figure legends was performed on non-normalized data.

Measurement of glutathione peroxidase activity. Glutathione peroxidase (GPx) activity was determined spectrophotometrically using a GPx assay kit from Cayman Chemicals (Ann Arbor, MI). The assay couples the reduction of peroxidized glutathione by GPx to the oxidation of $\mathrm{NADPH}$ to $\mathrm{NADP}^{+}$, which is accompanied by a decrease in absorbance at $340 \mathrm{~nm}$. The rate of decrease in the absorbance at $340 \mathrm{~nm}$ is directly proportional to the GPx activity in the samples.

In vivo detection of $\mathrm{O}_{2}{ }^{-}$using dihydroethidium. Dihyrdoethidium (DHE) was obtained from Invitrogen (Carlsbad, CA). To identify cellspecific superoxide formation in the brain in vivo, we used DHE as described previously (Quick and Dugan, 2001) with slight modifications. Briefly, mice were given two DHE intraperitoneal injections (final volume of $200 \mu \mathrm{l}, 27 \mathrm{mg} / \mathrm{kg}$ ) with a $30 \mathrm{~min}$ interval. At $18 \mathrm{~h}$ after the final injection of DHE, mice were anesthetized and then perfused with $4 \%$ paraformaldehyde. Brains were postfixed with $4 \%$ paraformaldehyde for $2 \mathrm{~d}$, and $25 \mu \mathrm{m}$ sections were cut on a cryostat. Slices were evaluated for ethidium fluorescence (excitation $\lambda, 488 \mathrm{~nm}$; emission $\lambda,>590 \mathrm{~nm}$ ) on a Zeiss (Oberkochen, Germany) LSM 510 confocal microscope system.

Measurement of protein carbonyls. Protein carbonyl content was measured in brain homogenates using 2,4-dinitrophenylhydrazine (DNPH) in a spectrophotometric assay (Levine et al., 1990). Briefly, sample tissues were sonicated in ice-cold homogenization buffer containing phosphatase and protease inhibitors (200 nM calyculin, $10 \mu \mathrm{g} / \mathrm{ml}$ leupeptin, 2 $\mu \mathrm{g} / \mathrm{ml}$ aprotinin, $1 \mathrm{~mm}$ sodium orthovanadate, and $1 \mu \mathrm{M}$ microcystinLR) and centrifuged at $1000 \times g$ for 15 min to sediment insoluble material. Three hundred microliter aliquots of the supernatant containing $0.7-1.5 \mathrm{mg}$ of protein were treated with $300 \mu \mathrm{l}$ of $10 \mathrm{~mm} \mathrm{DNPH}$, dissolved in $2 \mathrm{M} \mathrm{HCl}$, and compared with $2 \mathrm{M} \mathrm{HCl}$ alone (reagent blank). Duplicates were used for each sample. Samples then were incubated for $1 \mathrm{~h}$ at room temperature in the dark and stirred every $10 \mathrm{~min}$. Samples were precipitated with trichloroacetic acid (final concentration of $\sim 10 \%$ ) and centrifuged at $16,000 \times g$ at $4^{\circ} \mathrm{C}$ for $15 \mathrm{~min}$. The pellet was washed three times with $1 \mathrm{ml}$ of ethanol/ethyl acetate $(1: 1 \mathrm{v} / \mathrm{v})$. Each time, the pellet was lightly vortexed and left exposed to the washing solution for $10 \mathrm{~min}$ before centrifugation $(16,000 \times g$ for $5 \mathrm{~min})$. The final pellet was dissolved in $1 \mathrm{ml}$ of $6 \mathrm{M}$ guanidine in $10 \mathrm{~mm}$ phosphate buffer-trifluoroacetic acid, $\mathrm{pH} 2.3$, and the insoluble material was removed by centrifugation at $16,000 \times g$ for $5 \mathrm{~min}$. Absorbance was recorded at $366 \mathrm{~nm}$ for both DNPH-treated and HCl-treated samples. Protein carbonyl levels were calculated as nanomoles of carbonyl per milligram of protein using $\epsilon_{\max }=22,000 \mathrm{M} / \mathrm{cm}$, a molar absorbance coefficient for aliphatic hydrazones. Data were expressed as the percentage of protein carbonyl content from young wild-type samples. Protein carbonyl content also was examined via Western blots using an anti-2,3dinitrophenylhydrazone antibody from Chemicon.

Electrophysiology. Hippocampi from age-matched littermates were removed, and $400 \mu \mathrm{m}$ slices were prepared using a vibratome. The slices were perfused for $1-2 \mathrm{~h}$ with oxygenated artificial CSF (ACSF) (in mм: $125 \mathrm{NaCl}, 2.5 \mathrm{KCl}, 1.25 \mathrm{NaH}_{2} \mathrm{PO}_{4}, 25 \mathrm{NaHCO}_{3}, 25$ D-glucose, $2 \mathrm{CaCl}_{2}$, and $1 \mathrm{MgCl}_{2}$ ) in an interface tissue slice chamber at $30-32^{\circ} \mathrm{C}$. Pairedpulse facilitation (PPF), a presynaptic facilitation revealed when two stimuli at an intensity of $40-50 \%$ of the maximum extracellular field EPSP (fEPSP) were presented in rapid succession, was examined in these studies. PPF was measured by examining the ratio of the fEPSP slope of stimulus 2 to stimulus 1. Posttetanic potentiation (PTP) also was examined in these studies. PTP was induced with a single train of highfrequency stimulation (HFS) $(100 \mathrm{~Hz}$ for $1 \mathrm{~s})$ in the presence of APV (100 $\mu \mathrm{M})$ in ACSF. fEPSPs were recorded every $3 \mathrm{~s}$ for $3 \mathrm{~min}$ and were presented as the average of four individual traces. Finally, we also examined LTP in these studies. LTP was induced with either one train or four trains [ 5 min intertrain interval (ITI)] of $100 \mathrm{~Hz}$ HFS for $1 \mathrm{~s}$ after at least $20 \mathrm{~min}$ of stable baseline recordings. fEPSPs were recorded every $20 \mathrm{~s}$ and were presented as the average of four individual traces.

Behavioral studies. For all behavioral experiments, three groups of ECSOD transgenic mice and wild-type littermates at different ages were examined. The first group was 3-4 months of age, the second group was 13-14 months of age, and the third group was $>19$ months of age. Motor coordination and balance was tested using an accelerating rotating rod. Mice were tested repeatedly on 2 consecutive days, four trials per day, and the time for the mice to remain balanced on the rotating rod was recorded. Spatial learning and memory was tested using the Morris water maze. During the acquisition phase, mice were given four trials each day ( $60 \mathrm{~s}$ maximum, with an ITI of $1 \mathrm{~h}$ ) for 8 consecutive days. A probe trial was given at the end of the last training day to determine the type of search strategy developed by the mice. The number of platform crossings and the time spent in each quadrant was recorded in each probe trial. A visual platform task was given to 151 -year-old mice (wild-type, $n=9$; EC-SOD transgenic, $n=6$ ). The mice were given four trials (60 s maximum, ITI of $1 \mathrm{~h}$ ) each day for 2 consecutive days with the escape platform marked by a visible cue and moved randomly between four locations. The animals' trajectories were recorded with a video tracking system (EthoVision System; Noldus, Wageningen, The Netherlands). Associa- 
A

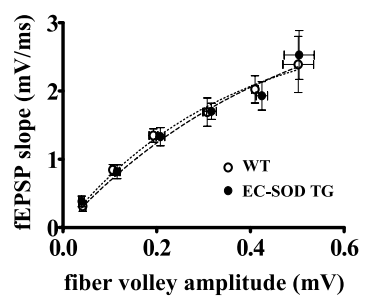

B
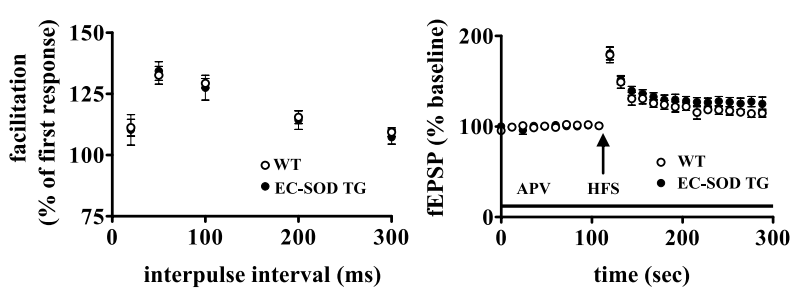

Figure 1. Baseline synaptic transmission and presynaptic plasticity are normal in aged EC-SOD transgenic mice. The 20-monthold mice were used in the experiments. $\boldsymbol{A}$, Plots of fEPSP slope versus fiber volley amplitudes. There was no significant difference between aged wild-type (WT) and aged EC-SOD transgenic (EC-SOD TG) mice in baseline synaptic transmission. Error bars indicate SEM for seven determinations. $\boldsymbol{B}$, Paired-pulse facilitation in aged WT and aged EC-SOD TG mice. Shown are responses to paired pulses in which the fEPSP slope of the response to the second stimulus was expressed as a percentage of the fEPSP slope of the response to the first stimulus plotted against the interpulse interval of the paired pulses. There was no significant difference between aged WT and aged EC-SOD TG mice. Error bars indicate SEM for six determinations. C, Posttetanic potentiation in aged WT and aged EC-SOD TG mice. Shown are points representing 10 responses measured before HFS and 15 responses measured immediately after HFS in the presence of the NMDA receptor antagonist APV $(100 \mu \mathrm{m})$. There was no significant difference between aged WT and aged EC-SOD TG mice by two-way ANOVA, followed by Bonferroni's post hoc tests. Error bars indicate SEM for five determinations for WT mice and four determinations for EC-SOD TG mice.

Young EC-SOD transgenic mice have impaired LTP, whereas aged EC-SOD transgenic mice exhibit an enhanced LTP compared with wild-type mice Hippocampal LTP has been proposed to be a cellular substrate for mammalian learning and memory. It has been demonstrated that superoxide can serve as a cellular messenger during LTP (Klann, 1998; Klann et al., 1998; Knapp and Klann, 2002b). Conversely, superoxide can be a toxic molecule that contributes to oxidative stress in neurons (Oury et al., 1992). Together, these previous findings suggested that a decrease in superoxide levels in EC-SOD transgenic mice might have different effects on LTP during the aging process. Therefore, we evaluated this form of synaptic plasticity in the mutant mice at 3 months of age and at 20 months of age and greater. fEPSPs were recorded in stratum radiatum in area CA1 of hippocampal slices in response to stimulation of the

tive memory was tested using the conditioned fear paradigm. The training sessions for contextual and cued fear conditioning consisted of a 2 min exploration period followed by two conditioned stimulus- unconditioned stimulus pairings separated by $1 \mathrm{~min}$ (footshock intensity of $0.75 \mathrm{~mA}, 2 \mathrm{~s}$ duration; tone of $90 \mathrm{db}$ white noise, $30 \mathrm{~s}$ duration). Context tests were performed in the training chamber after $24 \mathrm{~h}$. Cue tests were performed in a distinct chamber $25 \mathrm{~h}$ after training; baseline freezing was monitored ( 3 min duration) before presentation of the tone ( $90 \mathrm{db}$ white noise, 3 min duration).

Data analysis. The results are presented as mean \pm SEM. For comparisons between two groups, a two-tailed Student's $t$ test was used. All time course experiments involving multiple comparisons were analyzed using a two-way ANOVA, followed by individual post hoc tests when applicable. Error probabilities of $p<0.05$ were considered statistically significant.

\section{Results}

\section{Aged EC-SOD transgenic mice exhibit normal synaptic} transmission and presynaptic plasticity

We have shown previously that young EC-SOD transgenic mice have normal basal synaptic transmission and presynaptic transmission (Thiels et al., 2000). To determine whether this was the case in aged animals, we compared EC-SOD transgenic mice at 19 months of age or greater with their wild-type littermates. First, we tested the synaptic input-output relationship in area CA1 by eliciting synaptic responses with a range of stimulus intensities. We observed no significant differences between wild-type and EC-SOD transgenic mice (Fig. 1A). Second, we compared the PPF, which measures a calcium-dependent form of presynaptic plasticity in area CA1. Similar to input-output function, we observed no difference in PPF between the two genotypes (Fig. $1 B$ ). Finally, we tested PTP, which is manifested as a robust enhancement of synaptic efficacy that is presynaptic and observed after a brief period of HFS. We performed the experiments in the presence of APV (100 $\mu \mathrm{m})$, an NMDA receptor antagonist. As shown in Figure 1C, PTP in area CA1 was indistinguishable between hippocampal slices from wild-type and EC-SOD transgenic mice. Together, these results suggest that overexpression of EC-SOD does not exert an adverse effect on general synaptic function and two forms of presynaptic plasticity during aging.
Schaffer collateral/commissural pathway. In agreement with previous findings (Thiels et al., 2000), a single $100 \mathrm{~Hz}$ train evoked early-phase LTP (E-LTP) that was significantly impaired in 3 -month-old EC-SOD transgenic mice (119 $\pm 7 \%$ of baseline 30 min after HFS) compared with the wild-type controls (151 $\pm 6 \%$ of baseline $30 \mathrm{~min}$ after HFS) (Fig. 2A). Using the same E-LTP induction protocol, we found that aged EC-SOD transgenic mice had significantly increased LTP $(144 \pm 6 \%$ of baseline $30 \mathrm{~min}$ after HFS) compared with their aged wild-type littermates (125 \pm $5 \%$ of baseline $30 \mathrm{~min}$ after HFS) (Fig. 2 B). It is well known that multiple and spaced stimuli induce a very robust and long-lasting LTP, termed late-phase LTP (L-LTP). We therefore examined L-LTP induced by four trains of HFS with 5 min intertrain interval in aged wild-type and EC-SOD transgenic mice. Multiple trains of HFS produced very little L-LTP in slices from aged wildtype mice ( $111 \pm 7 \%$ of baseline 180 after the last train of HFS) (Fig. 2C). In striking contrast, robust L-LTP was induced in slices from aged EC-SOD transgenic mice (144 $\pm 8 \%$ of baseline 180 min after the last train of HFS) (Fig. 2C). Together, these findings strongly suggest that there is an age-related alteration in the role of superoxide in hippocampal LTP.

SODs convert superoxide to hydrogen peroxide and oxygen. Because hydrogen peroxide has been shown to improve LTP in aged wild-type mice (Kamsler and Segal, 2003b), we considered the possibility that the enhanced LTP in aged EC-SOD transgenic mice was caused by altered hydrogen peroxide metabolism. To examine this possibility, slices from aged wild-type and EC-SOD transgenic mice were given a single $100 \mathrm{~Hz}$ HFS in the presence of catalase $(260 \mathrm{U} / \mathrm{ml})$, an enzyme that catalyzes the reduction of hydrogen peroxide. Figure $2 D$ shows that LTP induced in the presence of catalase was still enhanced in aged EC-SOD transgenic mice (148 $\pm 16 \%$ of baseline 30 min after HFS) compared with LTP induced in the presence of catalase in wild-type controls ( $115 \pm 8 \%$ of baseline $30 \mathrm{~min}$ after HFS). It should be noted that catalase had no effect on the magnitude of LTP in slices from aged EC-SOD transgenic mice (Fig. 2B,D). Interestingly, addition of catalase caused a small but statistically significant decrease in LTP in slices from aged wild-type mice [without catalase, $125 \pm 5 \%$ of baseline $30 \mathrm{~min}$ after HFS (Fig. $2 \mathrm{~B}$ ); with catalase, $115 \pm 8 \%$ of baseline $30 \mathrm{~min}$ after HFS (Fig. 2D)]. These results indicate that 
the enhancement of LTP in aged EC-SOD transgenic mice was not caused by extra hydrogen peroxide produced by the overexpression of EC-SOD. These results also are consistent with the idea that hydrogen peroxide contributes to the expression of LTP in area CA1 of wild-type mice (Thiels et al., 2000; Kamsler and Segal, 2003a).

\section{Aged EC-SOD transgenic mice are protected against age-related decline in spatial memory}

Because hippocampal LTP may be a cellular substrate for hippocampus-dependent memory, our observations of improved LTP in area CA1 in aged EC-SOD transgenic mice lead us to the predict that hippocampus-dependent memory function also would be improved in these mice. To test this prediction, we examined the spatial learning and memory of wild-type and EC-SOD transgenic mice in the Morris water maze. We examined mice in three different age groups: 3-4 months of age, 13-14 months of age, and 19-20 months of age. During acquisition of the task, there were no differences with respect to escape latencies (Fig. 3A) and path length (data not shown) between the wildtype and EC-SOD transgenic mice at 3-4 months of age and 13-14 months of age, indicating that the wild-type and EC-SOD transgenic mice learn the platform position in a similar manner. However, at 19-20 months of age, the EC-SOD transgenic mice had significantly shorter escape latencies than the wild-type mice as determined by a two-way ANOVA (Fig. 3A). To assess spatial memory, we tested the mice in a probe trial during which the platform was removed from the pool and the mice were allowed to search for $60 \mathrm{~s} \mathrm{(} 1 \mathrm{~h}$ after the completion of the last training trial). We observed that, at 19-20 months of age, the EC-SOD transgenic mice spent significantly more time in the target quadrant than their wild-type littermates (Fig. 3C). Next, we compared the 3-monthold mice to the 19- to 20-month-old mice within each genotype. We found that young wild-type mice spent significantly more time in the target quadrant (Fig. $3 C$ ) than the old wild-type mice ( $p<0.01$ by Student's $t$ test). In contrast, we found no significant difference between the young and aged EC-SOD transgenic mice, which suggests that the EC-SOD transgenic mice did not exhibit an age-related decline in spatial memory. Consistent with this idea, when we examined the number of platform crossings, we found that the two older groups of EC-SOD transgenic mice tend to cross the original hidden platform place more often than their wildtype littermates (Fig. 3D). Altogether, these data demonstrate that overexpression ofEC-SOD protects mice against age-related impairments in hippocampus-dependent spatial memory.

To examine the possibility that the differences in spatial memory between the wild-type and EC-SOD transgenic mice was attributable to poor vision, motivation, or swimming ability, we tested naive mice ( 12 months of age) on a visible platform task in which mice learned to associate a distinct visible cue (a black pole) with an escape platform. The position of the platform and swim-start position varied from trial to trial. We found that there
C

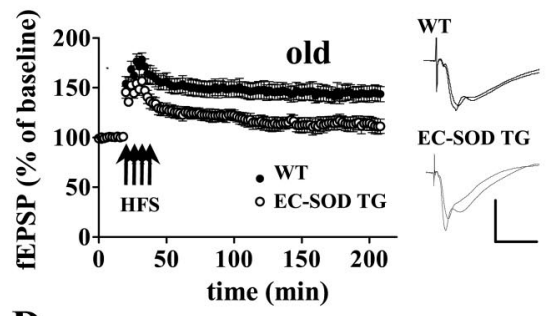

D

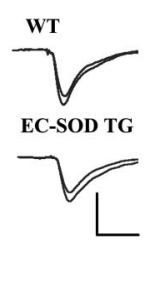

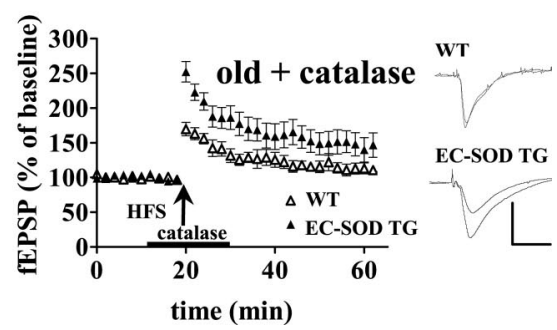

Figure 2. YoungEC-SOD transgenic mice have impaired LTP, whereas aged EC-SOD transgenic mice exhibit enhanced LTP when

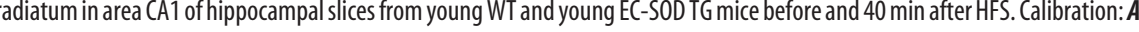
政 列 for $1 \mathrm{~s}$ ) in the presence of catalase $(260 \mathrm{U} / \mathrm{ml})$ as indicated by the bar ( $>20$-month-old WT, $n=6$; EC-SOD TG, $n=6)$. The traces shown on the right are representative fEPSPs recorded in stratum radiatum in area CA1 of hippocampal slices from aged WT and aged EC-SOD TG before and 40 min after HFS. In all panels, the cumulative data are plotted as mean \pm SEM.

were no differences in latencies to find the platform (Fig. 3B), swim speed, or distance traveled (data not shown) in this task. In addition, in open-field analysis, the wild-type and EC-SOD transgenic mice at all ages exhibited healthy locomotor abilities and exploratory behavior (data not shown). Thus, the overexpression of EC-SOD results in protection against age-related decline in performance in the Morris water maze by preventing age-related decline in spatial memory.

\section{Aged EC-SOD transgenic mice exhibit impaired associative memory}

We reported previously that contextual fear conditioning, a hippocampus and amygdala-dependent process, was significantly impaired in young EC-SOD transgenic mice (Thiels et al., 2000). Therefore, we tested whether there was an age-related alteration in contextual fear conditioning in 19- to 20-month-old EC-SOD transgenic mice. Figure $4 B$ shows that wild-type and EC-SOD transgenic mice exhibited comparably low levels of freezing during training (wild-type, $3.4 \pm 0.7 \%$ freezing; EC-SOD transgenic, $2.8 \pm 0.6 \%$ freezing). When tested for conditioning to the context $24 \mathrm{~h}$ after training, EC-SOD transgenic mice still exhibited significantly lower levels of freezing to the context than wild-type mice did (wild-type, $48 \pm 5 \%$ freezing; EC-SOD transgenic, $31 \pm$ $4 \%$ freezing) (Fig. 4 B), which was similar to the pattern observed in young wild-type and EC-SOD transgenic mice (wild-type, $42 \pm 5 \%$ freezing; EC-SOD transgenic, $19 \pm 4 \%$ freezing) (Fig. $4 A$ ). However, the aged EC-SOD transgenic mice did freeze more than the young EC-SOD transgenic mice. In contrast to contextual fear conditioning, when we examined cued conditioning, an 
A

- 3-4 month WT $\bullet$ 3-4 month TG

$\triangle$ 13-14month WT $\triangle 13-14$ month TG

口 19-20month WT 19-20month TG
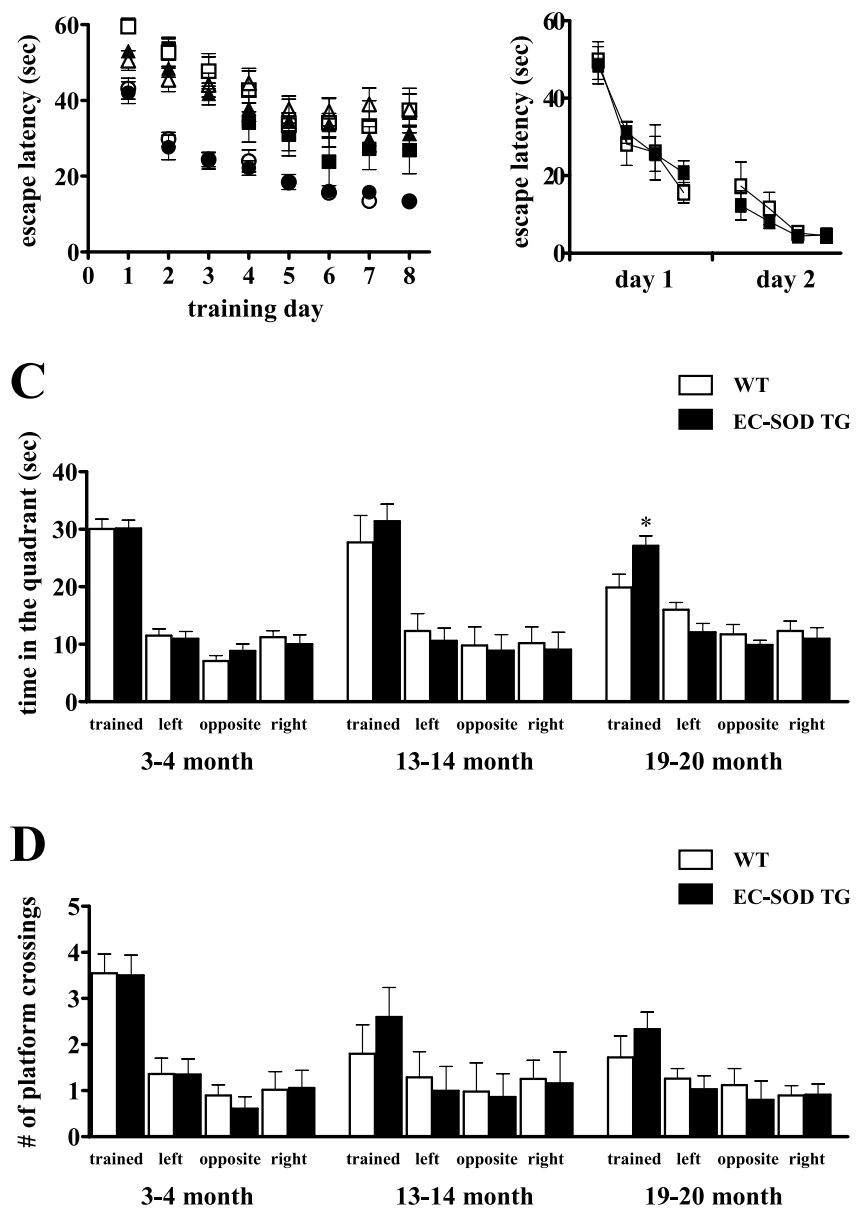

Figure 3. Aged EC-SOD transgenic mice do not exhibit a significant age-related decline in spatial memory in the Morris water maze. $\boldsymbol{A}$, Time spent searching for the platform on each training day. Data are plotted as mean \pm SEM. Wild-type (WT) and EC-SOD transgenic (EC-SOD TG) littermates were used. For each genotype: $3-4$ month group, $n=20 ; 13-14$ month group, $n=10 ; 19-20$ month group, $n=18$. $\boldsymbol{B}$, Visible platform test. No significant difference was observed between WT and EC-SOD TG. Data are plotted as mean \pm SEM. The 12-month-old mice were used for this test (WT, $n=9$; EC-SOD TG, $n=6$ ). C, Time spent in each of the quadrants in the probe trial on the last training day. ${ }^{*} p<0.05$ compared with aged wild-type mice by Student's $t$ test. The time spent in the target quadrant was not significantly different between wild-type and EC-SOD transgenic in the 3-4 month and 13-14 month groups. D, The number of platform crossings in the probe trial is shown for the target quadrant and the corresponding location in the other quadrants.. The 19- to 20-month-old EC-SOD TG mice tend to cross the original hidden platform place more often than the wild-type littermates. $p=0.1$ by Student's $t$ test.

amygdala-dependent memory process, we found that aged ECSOD transgenic mice exhibited freezing comparable with that of aged wild-type mice (Fig. $4 B$ ). In other behavioral tests, we found that aged wild-type and EC-SOD transgenic mice showed similar responses in the open field (an exploratory behavior test), the elevated plus maze (an anxiety measurement), and the hot plate (a pain threshold test), suggesting that there were no gross neurological abnormalities in these animals (data not shown). Collectively, these data indicate that, although contextual fear memory is compromised in EC-SOD transgenic mice regardless of age, the contextual fear memory in EC-SOD transgenic mice improves with age.
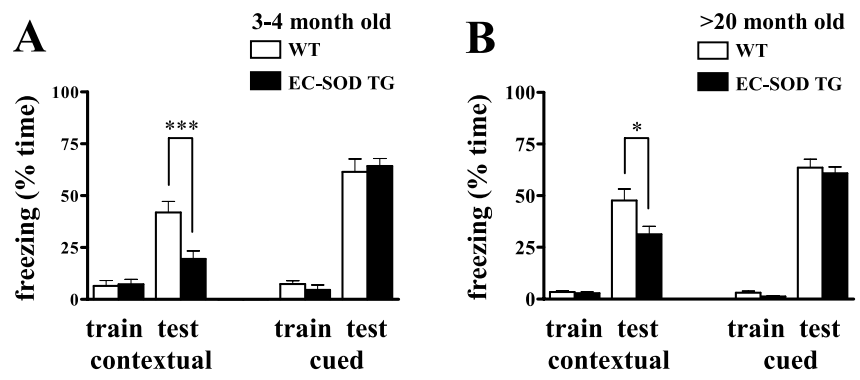

Figure 4. Impaired associative memory in EC-SOD transgenic mice. $\boldsymbol{A}$, Freezing behavior was expressed as the percentage of time during training (train) and testing (test) for 3-month-old wild-type (WT) and EC-SOD transgenic (EC-SOD TG) mice. Bar graphs on the left show the percentage of time that the mice froze to context $24 \mathrm{~h}$ after training. Bar graphs on the right show the percentage of time that the mice froze to the auditory cue $25 \mathrm{~h}$ after training. (WT, $n=7$; EC-SOD TG, $n=10$ ). Data are plotted as mean \pm SEM. ${ }^{* * *} p<0.001$ compared with aged wild-type mice by Student's $t$ test. $\boldsymbol{B}$, Similar to $\boldsymbol{A}$, except that aged ( $>20$ months of age) mice were used for this study (WT, $n=20$; EC-SODTG, $n=22$ ). ${ }^{*} p<0.05$ compared with aged wild-type mice by Student's $t$ test.

\section{Aged EC-SOD mice exhibit better motor coordination and motor learning}

Aged mice have been reported to have impaired motor skills (Forster et al., 1996). Therefore, we used the rotarod test to investigate whether EC-SOD overexpression could protect against age-related decline in motor coordination and motor learning. The wild-type and EC-SOD transgenic mice had similar body weight at each of the ages examined (Fig. $5 A$ ). Mice were trained four trials per day for $2 \mathrm{~d}$ to balance on an accelerating rod, and the time they remained on the rod in each trial was recorded. On the second training day, 3-month-old EC-SOD transgenic mice and their wild-type littermates had a comparable latency to fall (Fig. 5B). In contrast, the EC-SOD transgenic mice performed significantly better in both the 13-14 month age group and $>19$ month age group (Fig. 5B). These data indicate that mice that overexpress EC-SOD exhibit improved motor coordination during aging compared with their wild-type littermates.

With subsequent training, the latency at which wild-type and EC-SOD transgenic mice fell from the rotarod increased $(p<$ 0.0001, two-way ANOVA, effect of trial), indicating learning of motor skills. We therefore compared the rate of improvement on repeated trials as an index of motor learning ability. At 3 months of age, wild-type and EC-SOD transgenic mice learned at the same rate $(p=0.36$, ANCOVA; $p=0.85$, two-way ANOVA, effect of genotype) (Fig. 5C). In contrast, at 13 months of age and at $>19$ months of age, EC-SOD transgenic mice learned at a higher rate than their wild-type littermates $[\dagger p<0.0001$, ANCOVA; $\$ p<0.0001$, two-way ANOVA, effect of genotype) (Fig. $5 D, E)]$. In the $>19$-month-old group, Bonferroni's post hoc comparisons indicated a significant difference between wild-type and EC-SOD transgenic mice from trial 5 to trial $8\left({ }^{*} p<0.05\right.$, ${ }^{* *} p<0.001$, two-way ANOVA, followed by Bonferroni's post hoc tests). These findings suggest that EC-SOD overexpression improves motor learning in aged animals.

\section{EC-SOD overexpression reduces aging-related increases in superoxide levels in vivo}

EC-SOD expression in mouse brain has been described previously (Oury et al., 1999). The EC-SOD transgenic mice used in these studies have $\sim 10$-fold more hippocampal EC-SOD activity than wild-type mice, and the overexpression of human EC-SOD did not affect the levels of endogenous EC-SOD (Thiels et al., 2000). To determine whether EC-SOD overexpression alters the 
levels of other antioxidant enzymes, we performed Western blot analyses of SOD-1, SOD-2, and catalase and enzyme activity assays of GPx in hippocampal homogenates prepared from both young and old mice. These analyses revealed that human EC-SOD overexpression did not alter either of the other endogenous SODs (SOD-1 and SOD-2), catalase, or GPx activity in the hippocampus (Table 1). We did observe a nonstatistically significant aging-related increase in SOD-2 and a statistically significant aging-related increase in SOD-1, catalase, and GPx activity in wild-type and aged EC-SOD transgenic mice (Table 1). We proceeded to examine whether EC-SOD overexpression could reduce in vivo superoxide levels in the transgenic mice by using DHE, a superoxide-sensitive dye that has been used to localize superoxide in tissues $(\mathrm{Mu}-$ rakami et al., 1998). Fluorescence reflecting DHE oxidation to ethidium is stable to fixation procedures (Quick and Dugan, 2001). We assessed the level of tissue autofluorescence in slices from wild-type and EC-SOD transgenic mice that received no DHE and found that background tissue fluorescence was very low and did not differ between wild-type and EC-SOD transgenic mice (data not shown). We then compared sections prepared from 3-month-old or 20-monthold wild-type and EC-SOD transgenic mice that received two intraperitoneal injections of DHE (see Materials and Methods). Shown in Figure 6 is ethidium fluorescence in the whole hippocampus (Fig. $6 A$ ), hippocampal area CA1 (Fig. $6 B$, first row), hippocampal area CA3 (Fig. $6 B$, second row), and the cerebellum (Fig. $6 B$, third row). In each area examined, superoxide levels were increased in aged mice compared with young mice. In area CA1 young EC-SOD transgenic mice exhibited lower levels of superoxide compared with young wild-type mice (Fig. 6C). In addition, the age-related increases in superoxide in wild-type mice were substantially decreased in aged EC-SOD transgenic mice (Fig. 6C). Examination of other brain regions showed similar patterns of superoxide levels as were measured in hippocampal CA1. These results suggest that there is an age-related increase in superoxide levels in the hippocampus and that this increase can be prevented by overexpression of EC-SOD.

\section{A}

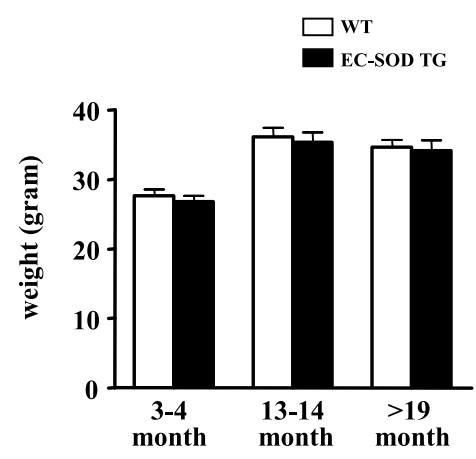

B

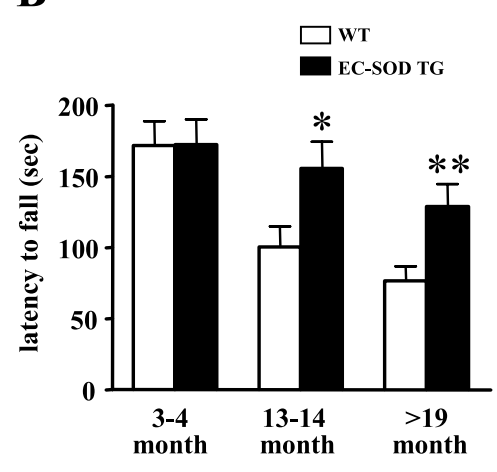

C
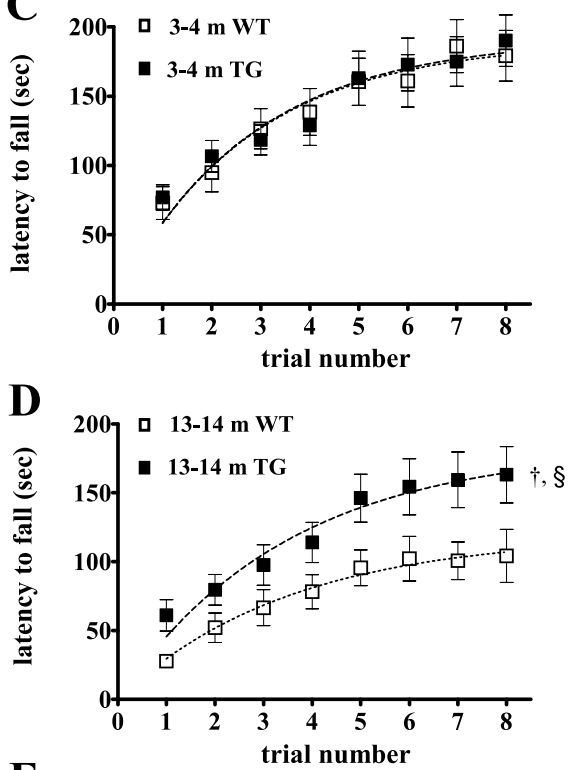

$\mathbf{E}$

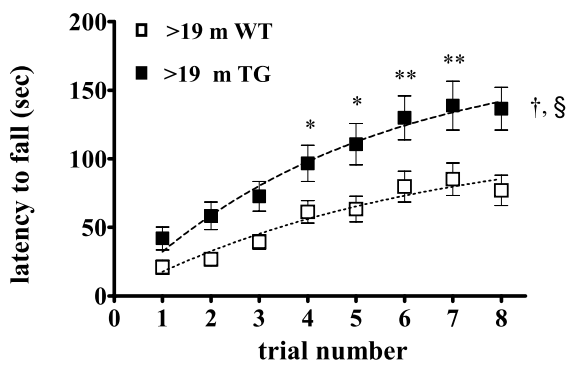

Figure 5. Aged EC-SOD transgenic mice exhibit better motor performance than wild-type littermates. $A$, Body weight of wild-type mice (WT) and EC-SOD transgenic mice (EC-SOD TG). B, Motor coordination accessed by the rotating rod test. The time for the mice to remain on the rod on the second training day was plotted. Aged EC-SOD TG mice performed significantly better than wild-type mice. Data are plotted as mean \pm SEM (3-4 month group, $n=20 ; 13-14$ month group, $n=15 ;>19$ month group, $n=20) .{ }^{*} p<0.05,{ }^{* *} p<0.01$ compared with age-matched wild-type mice by Student's $t$ test. $C-E$, Motor learning ability was assessed by measuring the rate of improvement after repeated trials in the rotating rod test. The time for the mice to remain on the rod in each trial was plotted as mean \pm SEM. Data were transformed using $\log 10$, and then a test for homogeneity of slopes (ANCOVA) was performed to determine whether the slopes were parallel. At 13 months of age and at $>19$ months of age, EC-SOD transgenic mice learned at a higher rate than their wild-type littermates $\left({ }^{\dagger} p<0.0001\right.$, ANCOVA; $^{\S} p<0.0001$, two-way ANOVA, effect of genotype). In the $>19$-month-old group, Bonferroni's post hoc comparisons indicated a significant difference between wild-type and EC-SOD transgenic mice from trial 5 to trial $8\left({ }^{*} p<0.05,{ }^{* *} p<0.001\right.$, two-way ANOVA, followed by Bonferroni's posthoc tests).

Table 1. Measurement of antioxidants: SOD-1, SOD-2, catalase, and GPx from hippocampal tissues of EC-SOD transgenic mice and wild-type littermates

\begin{tabular}{|c|c|c|c|c|}
\hline & \multicolumn{2}{|l|}{3 months old } & \multicolumn{2}{|l|}{$>20$ months old } \\
\hline & Non- $\operatorname{Tg}(n)$ & EC-SOD Tg $(n)$ & Non- $\operatorname{Tg}(n)$ & EC-SOD Tg $(n)$ \\
\hline SOD-1 & $1.00 \pm 0.11(4)$ & $1.08 \pm 0.08(4)$ & $1.27 \pm 0.14^{*}(6)$ & $1.24 \pm 0.08^{*}(5)$ \\
\hline SOD-2 & $1.00 \pm 0.06(4)$ & $1.04 \pm 0.05(6)$ & $1.19 \pm 0.16(4)$ & $1.18 \pm 0.07(5)$ \\
\hline Catalase & $1.00 \pm 0.07(5)$ & $1.02 \pm 0.06(5)$ & $1.25 \pm 0.04^{* *}(5)$ & $1.27 \pm 0.07^{* *}(5)$ \\
\hline GPx & $11.3 \pm 0.73(4)$ & $11.6 \pm 0.67(4)$ & $16.6 \pm 0.64^{* *}(7)$ & $15.8 \pm 0.72^{* *}(7)$ \\
\hline
\end{tabular}

Hippocampal homogenates prepared from wild-type and EC-SOD transgenic (Tg) mice were examined by Western blot analysis with antibodies for SOD-1, SOD-2, and catalase. In addition, enzymatic activity of GPX was measured. Results of SOD-1, SOD-2, and catalase are presented as arbitrary units determined by densitometry and normalized to 3-month-old wild-type controls. GPx activity was measured as the rate of NADPH consumption (in nanomoles) per minute per milligram of protein. The number of determinations for each measurement is indicated in parentheses. Data are presented as mean \pm SEM. Two-way ANOVA followed by Bonferroni's post hoc tests showed that there is no interaction between age and genotype, and no effect of genotype was observed. However, there was an effect of age for SOD- 1 and catalase levels and GPx activity ( ${ }^{*} p<0.05$ and ${ }^{* *} p<0.005$, respectively). There also was a nonstatistically significant trend for an age effect for SOD-2 ( $p=0.077)$. Thus, antioxidant activity was not altered in EC-SOD transgenic mice, but overall antioxidant levels increase with age in both wild-type and EC-SOD transgenic mice.

EC-SOD overexpression decreases protein carbonyl levels in aged mice

As a first step to determine whether EC-SOD overexpression could reduce brain levels of oxidative damage, we measured brain protein carbonyl levels as an index of protein oxidation. First, we measured protein carbonyl levels in brain homogenates using a spectrophotometric assay. We found that there was no difference between young wild-type mice $(11.1 \pm 2.6 \mathrm{nmol}$ carbonyl/mg 
A

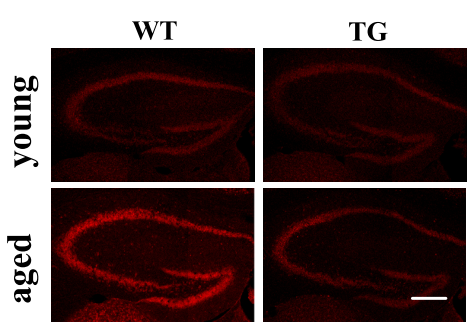

B

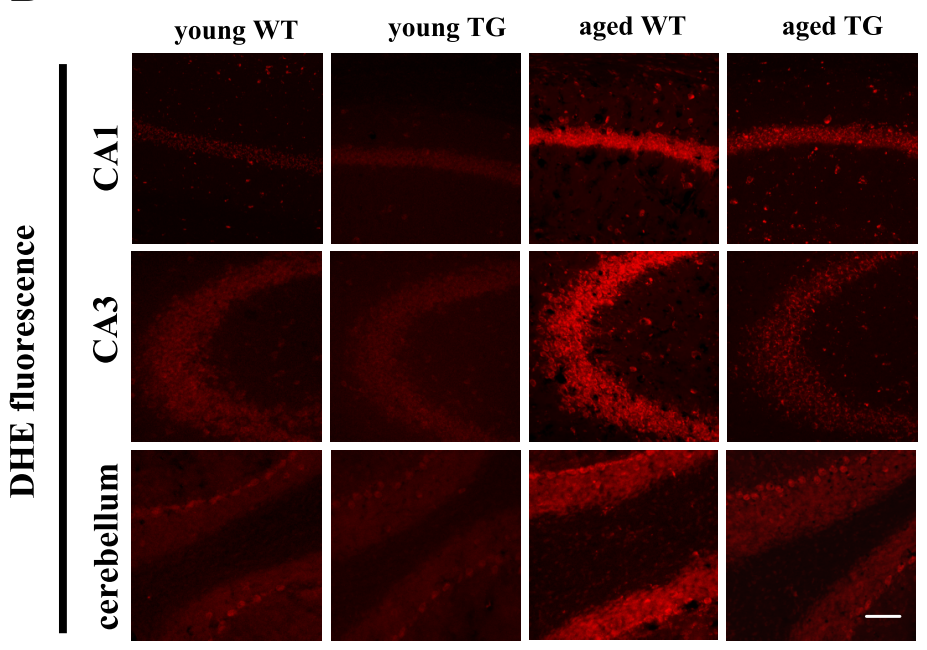

Figure 6. Endogenous levels of superoxide in young and old wild-type and EC-SOD transgenic mice. Brain sections were prepared from four 3-month-old (young) and four 20-month-old (aged) wild-type (WT) and EC-SOD transgenic (TG) mice that received two intraperitoneal injections of DHE (for details, see Materials and Methods). Mice that were not injected with DHE were used as controls for autofluorescence. $\boldsymbol{A}$, Coronal sections of the hippocampus showing differences in ethidium fluorescence between young and aged WT and young and aged TG mice. Scale bar, $300 \mu \mathrm{m}$. B, Coronal sections through CA1 and CA 3 of the hippocampus and cerebellum showing differences in ethidium fluorescence. The images were taken with the same settings, and a typical image for each region is shown. Scale bar, $75 \mu \mathrm{m}$. C, Quantification of fluorescence in hippocampal area CA1. DHE fluorescence was measured with Scion Image software, using 1 inch square throughout the soma of CA1 pyramidal cells from at least eight sections. Data were graphed as pixel intensity, mean $\pm \mathrm{SEM} ; n>40$. Tissue autofluorescence (control) from mice that received no DHE is shown by the left bar. Values were compared by one-way ANOVA, followed by Tukey's post hoc tests, indicating significantly enhanced levels in aged mice compared with young mice $\left.{ }^{* *} p<0.01\right)$. Student's $t$ test showed significant differences between young wild-type (young WT) and young transgenic (young TG) ${ }^{\sharp} p<0.05$ ), as well as between aged wild-type (aged WT) and aged transgenic (aged TG) ${ }^{\# \# \# ~} p<0.0001$ ) mice.

protein) and young EC-SOD transgenic mice $(12.1 \pm 2.7 \mathrm{nmol}$ carbonyl/mg protein) (Fig. $7 A$ ). In contrast, we observed an agerelated increase in protein carbonyl content in wild-type mice (16.3 $\pm 4.0 \mathrm{nmol}$ carbonyl/mg protein) that was significantly decreased in EC-SOD transgenic mice (13.9 $\pm 4.2 \mathrm{nmol}$ carbonyl/mg protein). We proceeded to use an anti-2,3dinitrophenylhydrazone antibody to detect protein carbonyls in brain homogenates by Western blot analysis. Again, we found that the aged EC-SOD mice had reduced protein carbonyl levels compared with their wild-type counterparts (Fig. $7 B$ ). It should be noted that young EC-SOD transgenic mice appear to exhibit increased protein carbonyl levels compared with young wild-type mice (Fig. $7 B$ ), perhaps attributable to increases in hydrogen peroxide produced by EC-SOD overexpression. Regardless, these findings strongly suggest that overexpression of EC-SOD decreases aging-related enhancement in protein carbonyl levels in the brain, thereby decreasing oxidative damage associated with aging.

\section{Effects of EC-SOD overexpression on} MAP kinases

c-Jun N-terminal kinase (JNK), p38 kinase, and ERK belong to the mitogenactivated protein kinase family whose members are known to be involved in a wide variety of cellular stresses, including oxidative stress and apoptosis. We hypothesized that, by reducing the oxidative stress in neurons of aged animals, ECSOD would decrease the levels of active MAPKs. To test this hypothesis, we examined the total levels and the levels of active MAP kinases in the hippocampus on Western blots. We found that the total levels of all three MAPKs were not changed during aging in the wild-type and ECSOD transgenic mice (data not shown). The levels of the active, phosphorylated forms of JNK2 and JNK3 also showed no differences with respect to either age or genotype (data not shown). We did observe an age-related increase in the levels of the active, phosphorylated forms of both p38 (Fig. 8A) and ERK2 (Fig. $8 B$ ) in hippocampal homogenates from wild-type mice. In contrast, the age-related increases in active p 38 and active ERK2 were absent in hippocampal homogenates from the ECSOD transgenic mice (Fig. $8 A, B$ ). These data indicate that overexpression of ECSOD prevents age-related increases in active p38 and active ERK2 in the hippocampus.

\section{Discussion}

EC-SOD overexpression enhances LTP and memory in aged mice

In this study, we used a series of electrophysiological, behavioral, and biochemical analyses to investigate the role played by superoxide in the nervous system during aging using mice that overexpress ECSOD as a model system. We examined hippocampal LTP and memory-related behavioral performance over the lifespan of EC-SOD transgenic mice and their wild-type littermates to understand how superoxide regulates these processes during aging. In previous studies, we showed that young EC-SOD transgenic mice had normal synaptic transmission and presynaptic plasticity but impaired LTP in hippocampal area CA1 (Thiels et al., 2000), suggesting a positive role of superoxide as part of the biochemical signaling required for this type of synaptic plasticity. In the present studies, we found that aged EC-SOD transgenic mice exhibit improved E-LTP and L-LTP compared with their wild-type littermates (Fig. $2 B, C$ ), uncovering an age-dependent alteration in the function of superoxide. Consistent with our results, similar changes in hippocampal LTP also have been reported in transgenic mice overexpressing SOD-1 (Kamsler and Segal, 2003b). Based on all of these findings, we propose that superoxide has dual roles with respect to LTP that depend on age. In young wild-type mice, physiological production of superoxide is a necessary component of LTP. In young EC-SOD transgenic mice, SOD overexpression scavenges these 
A

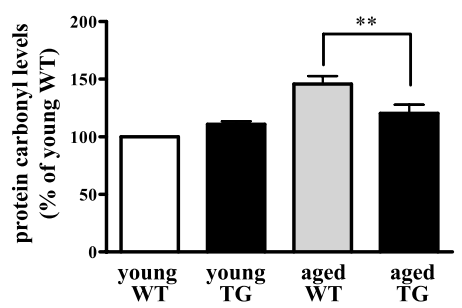

B

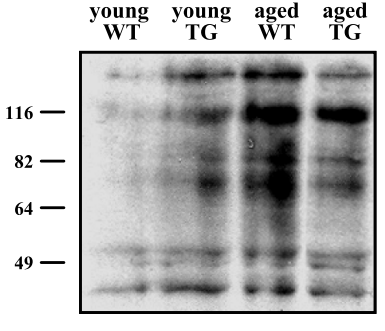

Figure 7. Decreased brain protein oxidation in aged EC-SOD transgenic mice. A, Spectrophotometric assays for protein carbonyls in brain homogenates. Levels of protein oxidation (protein carbonyls) were determined as described in Materials and Methods. Results were expressed as percentage of young wild-type (young WT) values. Error bars indicate SEM for five determinations. In this assay, young EC-SOD transgenic mice (young TG) exhibited similar protein carbonyl levels compared with young WT mice, whereas old EC-SOD transgenic mice (aged TG) exhibited significantly lower protein carbonyl levels than old wild-type mice (aged WT); ${ }^{* *} p<0.05$ by Student's $t$ test. $\boldsymbol{B}$, Western blot analysis for protein carbonyls in hippocampal CA1 homogenates. The hippocampal CA1 region of young (3 months) or old (>20 months) wild-type or EC-SOD transgenic mice was dissected and homogenized, and the lysates were centrifuged to remove insoluble material. Total protein $(50 \mu \mathrm{g})$ was loaded in each lane. The experiment was performed four times; a representative blot is shown.

A

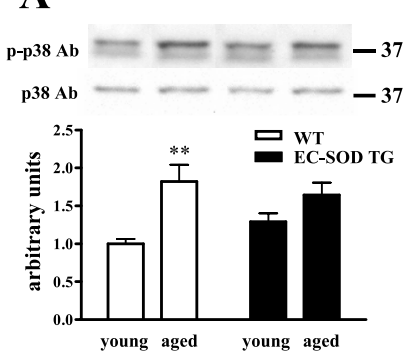

B
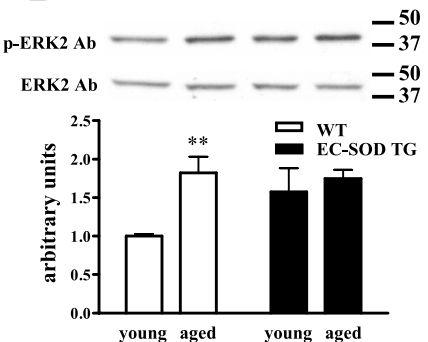

Figure 8. Decreased activation of p38 and ERK2 in the hippocampus of aged EC-SOD transgenic mice. Activation of p38 $(\boldsymbol{A})$ and ERK2 $(\boldsymbol{B})$ was assessed via Western blot analysis of phospho-p38 and phospho-ERK2 in hippocampal homogenates prepared from wild-type (WT) and EC-SOD transgenic (EC-SOD TG) mice at 3 months (young) and 20 months (aged) of age. Representative Western blots for phosphorylated p38 and total p38 and phosphorylated ERK2 and total ERK2 are shown at the top of each panel. For both p38 and ERK2, phosphoimmunoreactivity was normalized to total immunoreactivity, and results were plotted as arbitrary units compared with young wild-type controls. Values are mean \pm SEM for six determinations. ${ }^{* *} p<0.01$ compared with the same genotype by Student's $t$ test. Ab, Antibody.

physiological levels of superoxide and thereby impairs LTP. In aged transgenic mice, however, the baseline levels of superoxide are elevated (Fig. 6), which is sufficient to permit LTP expression despite the excess SOD. Conversely, because baseline superoxide levels are reduced in aged SOD-overexpressing mice, there is a partial protection against oxidative damage (Fig. 7), and, subsequently, the aged transgenic mice display more robust LTP than the wild-type littermates.

It is interesting that the overexpression of EC-SOD results in such profound intracellular effects that impact LTP. However, endogenous EC-SOD appears to be localized primarily in intracellular vesicle-like structures (Oury et al., 1999), and the human EC-SOD overexpressed in the transgenic mice appear to have a similar localization (Thiels et al., 2000). Thus, the decrease in intracellular superoxide we observed in the EC-SOD transgenic mice (Fig. 6) could be attributable to intracellular actions of ECSOD. Alternatively, if EC-SOD is present extracellularly, it could reduce intracellular superoxide by acting as an extracellular sink to pull superoxide out of neurons. Finally, superoxide may function as a transcellular messenger in LTP because it has been

shown that cell-impermeable superoxide scavengers strongly attenuate LTP (Klann et al., 1998).

In addition to impaired LTP, behavioral deficiencies in learning and memory tasks have been reported in aging rodents (Barnes, 2003). In our studies, several interesting findings emerged from the behavioral analyses. A standard behavioral test battery was used to assess the behavior of wild-type and EC-SOD transgenic mice at different ages. We found that young adult EC-SODoverexpressing mice performed normally in all of the behavioral tests, with the exception of deficits in contextual fear conditioning when compared with young adult wild-type littermates. However, the middle-aged (13-14 months old) and the old ( $>19$ months old) EC-SOD transgenic mice had substantially better performance in the Morris water maze (Fig. 3) and the rotarod test (Fig. 5), suggesting that EC-SOD overexpression improved both spatial memory and motor learning. In agreement with our findings, it was demonstrated recently that aged EC-SOD transgenic mice had significantly higher choice accuracy in the radialarm maze task than the wild-type controls (Levin et al., 2005), which also is consistent with improved spatial learning and memory. Interestingly, we found that the impairment in contextual memory deficits exhibited by young EC-SOD transgenic mice that were reported previously (Thiels et al., 2000) became less obvious as the mice aged, although the deficits could not be reversed completely (Fig. 4). This suggests the intriguing possibility that the role of superoxide as a signaling molecule is still dominant for this particular type of learning and memory. It will be of interest to determine why EC-SOD overexpression can protect against age-related impairments in water maze and radial-arm maze spatial memory but not contextual memory triggered by fear conditioning. Perhaps the regions of hippocampus that require ROS such as superoxide differ in these two types learning. This possibility remains to be determined.

It has been speculated that SODs, which lack catalase activity, may actually increase cellular damage by enhancing the local concentration of hydrogen peroxide. Consistent with this idea, we found that protein carbonyl levels in the hippocampus appear to be increased in young EC-SOD transgenic mice (Fig. 7B). Thus, high concentrations of hydrogen peroxide should be detrimental to synaptic plasticity, and there is evidence that high concentrations of exogenous hydrogen peroxide cause impairments in LTP (Pellmar et al., 1991; Auerbach and Segal, 1997; Watson et al., 2002; Kamsler and Segal, 2003a,b). However, hydrogen peroxide has been suggested to play a role in the expression of LTP (Thiels et al., 2000; Kamsler and Segal, 2003a). In wild-type mice, catalase prevents the full expression of LTP (Thiels et al., 2000), and low physiological concentrations of hydrogen peroxide enhance LTP (Kamsler and Segal, 2003a). Thus, hydrogen peroxide has paradoxical effects on LTP in young animals that are concentration dependent.

Hydrogen peroxide also can modulate LTP in mice that overexpress SOD-1 (Kamsler and Segal, 2003b). Young adult mice that overexpress SOD-1 were shown to have deficient LTP that can be reversed by hydrogen peroxide (Kamsler and Segal, 2003b). In the same study, aged SOD-1 transgenic mice exhibit normal LTP similar to young wild-type mice, whereas aged wild-type mice exhibit impaired LTP similar to young SOD-1 transgenic mice. Intriguingly, exogenous hydrogen peroxide reversed the deficiency in LTP in the aged wild-type mice but impaired LTP in the aged SOD-1 transgenic mice (Kamsler and Segal, 2003b). We found that the enhancement in LTP in aged EC-SOD transgenic mice was not impacted by catalase (Fig. 2D) and that the aged EC-SOD transgenic mice had lower protein carbonyl levels (Fig. 7), suggesting that EC-SOD overexpres- 
sion in fact reduced oxidative stress in aged animals. These findings suggest that, in contrast to SOD-1 transgenic mice, hydrogen peroxide does not contribute to age-related alterations in LTP in EC-SOD transgenic mice.

\section{EC-SOD overexpression reduces age-related increases in superoxide and markers of oxidative stress}

In vitro measurement of ROS is likely to alter the redox state of the tissue; therefore, we used a superoxide-sensitive dye to detect relative superoxide levels in vivo (Quick and Dugan, 2001). We observed an age-related increase in superoxide in the hippocampus that was significantly reduced in EC-SOD transgenic mice (Fig. 6). This suggests that EC-SOD overexpression indeed decreased the levels of superoxide in the hippocampus and thereby may have decreased oxidative stress in these mice. Consistent with this idea, we observed that age-related increases in protein carbonyl levels in the hippocampus were reduced in the EC-SOD transgenic mice (Fig. 7). The decreases in superoxide and protein carbonyls in the aged EC-SOD transgenic mice appear to be specific to EC-SOD because we observed that several antioxidant enzymes were not altered by overexpression of EC-SOD, although the overall levels of these enzymes increased with age in both wild-type and EC-SOD transgenic mice (Table 1). Such an increase in the antioxidant defense system could be an adaptation to deal with oxidative stress associated with aging.

Given the potentially harmful effects of oxidative stress, cell survival depends on the cellular response to the activation of stress signaling pathways. MAP kinases, including ERK, JNK, and p38, are among the major stress signaling pathways activated in response to oxidative stress (for review, see Martindale and Holbrook, 2002). Increased p38 activity has been reported in the aged rat brain (Martin et al., 2002), and age-related activation of JNK and p38 activation have been shown to result in apoptosis (O'Donnell et al., 2000) and hence are potentially involved in cell-fate determination under situations of oxidative stress (Guyton et al., 1996). Compared with their wild-type littermates, the aged EC-SOD transgenic mice did not exhibit a significant age-related increase in active p38 and ERK2 (Fig. 8). Thus, our results support the idea that EC-SOD overexpression can alleviate oxidative stress, thereby resulting in decreased activation of $\mathrm{p} 38$ and ERK2.

In summary, we have demonstrated that superoxide function in synaptic plasticity and memory shifts from a positive role to a negative role during aging. Although physiological levels of superoxide are necessary for hippocampal synaptic plasticity and associative memory when animals are young, in a chronic manner, superoxide plays a neurotoxic role during aging that likely contributes to brain dysfunction. Enhanced expression of ECSOD, therefore, can protect synaptic plasticity and learning and memory against oxidative damage during the aging process.

\section{References}

Auerbach JM, Segal M (1997) Peroxide modulation of slow onset potentiation in rat hippocampus. J Neurosci 17:8695-8701.

Barnes CA (2003) Long-term potentiation and the ageing brain. Philos Trans R Soc Lond B Biol Sci 358:765-772.

Esposito F, Ammendola R, Faraonio R, Russo T, Cimino F (2004) Redox control of signal transduction, gene expression and cellular senescence. Neurochem Res 29:617-628.

Forster MJ, Dubey A, Dawson KM, Stutts WA, Lal H, Sohal RS (1996) Agerelated losses of cognitive function and motor skills in mice are associated with oxidative protein damage in the brain. Proc Natl Acad Sci USA 93:4765-4769.

Gahtan E, Auerbach JM, Groner Y, Segal M (1998) Reversible impairment of long-term potentiation in transgenic $\mathrm{Cu} / \mathrm{Zn}$-SOD mice. Eur J Neurosci $10: 538-544$.
Guyton KZ, Liu Y, Gorospe M, Xu Q, Holbrook NJ (1996) Activation of mitogen-activated protein kinase by $\mathrm{H} 2 \mathrm{O} 2$. Role in cell survival following oxidant injury. J Biol Chem 271:4138-4142.

Halliwell B (1992) Reactive oxygen species and the central nervous system. J Neurochem 59:1609-1623.

Kamsler A, Segal M (2003a) Hydrogen peroxide modulation of synaptic plasticity. J Neurosci 23:269-276.

Kamsler A, Segal M (2003b) Paradoxical actions of hydrogen peroxide on long-term potentiation in transgenic superoxide dismutase-1 mice. J Neurosci 23:10359-10367.

Klann E (1998) Cell-permeable scavengers of superoxide prevent long-term potentiation in hippocampal area CA1. J Neurophysiol 80:452-457.

Klann E, Roberson ED, Knapp LT, Sweatt JD (1998) A role for superoxide in protein kinase $\mathrm{C}$ activation and induction of long-term potentiation. J Biol Chem 273:4516-4522.

Klivenyi P, St Clair D, Wermer M, Yen HC, Oberley T, Yang L, Flint Beal M (1998) Manganese superoxide dismutase overexpression attenuates MPTP toxicity. Neurobiol Dis 5:253-258.

Knapp LT, Klann E (2002a) Role of reactive oxygen species in hippocampal long-term potentiation: contributory or inhibitory? J Neurosci Res 70:1-7.

Knapp LT, Klann E (2002b) Potentiation of hippocampal synaptic transmission by superoxide requires the oxidative activation of protein kinase C. J Neurosci 22:674-683.

Levin ED, Christopher NC, Crapo JD (2005) Memory decline of aging reduced by extracellular superoxide dismutase overexpression. Behav Genet 35:447-453.

Levine RL, Garland D, Oliver CN, Amici A, Climent I, Lenz AG, Ahn BW, Shaltiel S, Stadtman ER (1990) Determination of carbonyl content in oxidatively modified proteins. Methods Enzymol 186:464-478.

Liu R, Liu IY, Bi X, Thompson RF, Doctrow SR, Malfroy B, Baudry M (2003) Reversal of age-related learning deficits and brain oxidative stress in mice with superoxide dismutase/catalase mimetics. Proc Natl Acad Sci USA 100:8526-8531.

Martin DS, Lonergan PE, Boland B, Fogarty MP, Brady M, Horrobin DF, Campbell VA, Lynch MA (2002) Apoptotic changes in the aged brain are triggered by interleukin-1beta-induced activation of p38 and reversed by treatment with eicosapentaenoic acid. J Biol Chem 277:34239-34246.

Martindale JL, Holbrook NJ (2002) Cellular response to oxidative stress: signaling for suicide and survival. J Cell Physiol 192:1-15.

Murakami K, Kondo T, Kawase M, Li Y, Sato S, Chen SF, Chan PH (1998) Mitochondrial susceptibility to oxidative stress exacerbates cerebral infarction that follows permanent focal cerebral ischemia in mutant mice with manganese superoxide dismutase deficiency. J Neurosci 18:205-213.

Nicolle MM, Gonzalez J, Sugaya K, Baskerville KA, Bryan D, Lund K, Gallagher M, McKinney M (2001) Signatures of hippocampal oxidative stress in aged spatial learning-impaired rodents. Neuroscience 107:415-431.

O'Donnell E, Vereker E, Lynch MA (2000) Age-related impairment in LTP is accompanied by enhanced activity of stress-activated protein kinases: analysis of underlying mechanisms. Eur J Neurosci 12:345-352.

Oury TD, Ho YS, Piantadosi CA, Crapo JD (1992) Extracellular superoxide dismutase, nitric oxide, and central nervous system $\mathrm{O}_{2}$ toxicity. Proc Natl Acad Sci USA 89:9715-9719.

Oury TD, Card JP, Klann E (1999) Localization of extracellular superoxide dismutase in adult mouse brain. Brain Res 850:96-103.

Pellmar TC, Hollinden GE, Sarvey JM (1991) Free radicals accelerate the decay of long-term potentiation in field CA1 of guinea-pig hippocampus. Neuroscience 44:353-359.

Quick KL, Dugan LL (2001) Superoxide stress identifies neurons at risk in a model of ataxia-telangiectasia. Ann Neurol 49:627-635.

Sun J, Folk D, Bradley TJ, Tower J (2002) Induced overexpression of mitochondrial Mn-superoxide dismutase extends the life span of adult Drosophila melanogaster. Genetics 161:661-672.

Thiels E, Urban NN, Gonzalez-Burgos GR, Kanterewicz BI, Barrionuevo G, Chu CT, Oury TD, Klann E (2000) Impairment of long-term potentiation and associative memory in mice that overexpress extracellular superoxide dismutase. J Neurosci 20:7631-7639.

Watson JB, Khorasani H, Persson A, Huang KP, Huang FL, O’Dell TJ (2002) Age-related deficits in long-term potentiation are insensitive to hydrogen peroxide: coincidence with enhanced autophosphorylation of $\mathrm{Ca}^{2+}$ / calmodulin-dependent protein kinase II. J Neurosci Res 70:298-308. 